\title{
Modeling electrical dispersion phenomena in Earth materials
}

\author{
Domenico Patella \\ Dipartimento di Fisica, Università degli Studi di Napoli «Federico II», Napoli, Italy
}

\begin{abstract}
It is illustrated that IP phenomena in rocks can be described using conductivity dispersion models deduced as solutions to a 2nd-order linear differential equation describing the motion of a charged particle immersed in an external electrical field. Five dispersion laws are discussed, namely: the non-resonant positive IP model, which leads to the classical Debye-type dispersion law and by extension to the Cole-Cole model, largely used in current practice; the non-resonant negative IP model, which allows negative chargeability values, known in metals at high frequencies, to be explained as an intrinsic physical property of earth materials in specific field cases; the resonant flat, positive or negative IP models, which can explain the presence of peak effects at specific frequencies superimposed on flat, positive or negative dispersion spectra.
\end{abstract}

Key words induced polarization - electrical dispersion spectra - geophysical applications

\section{Introduction}

Electric dispersion in rocks is the phenomenology on which the Frequency-Domain (FD) Induced Polarization (IP) geophysical survey method is based. FD IP effects are mostly investigated in mining, environmental and geothermal exploration. IP is the evidence that a complex mechanism of electrical conduction occurs in earth materials, necessitating an extension of the concept of resistivity, which in the FD can no longer be retained independent of frequency.

Many empirical models have been proposed to explain IP effects in rocks. Extended treatments can be found in the review books by Wait (1959), Bertin and Loeb (1976), Sumner (1976)

Mailing address: Prof. Domenico Patella, Dipartimento di Fisica, Università degli Studi di Napoli «Federico II», Napoli, Italy; e-mail: patella@na.infn.it and Fink et al. (1990). However, as pointed out by Wait (1982), the characterization of IP by empirical laws is vague and confusing, since, fundamentally, non-physical descriptions have been employed. To overcome this conceptual drawback, a generalized physical model has been examined in a very recent paper (Patella, 2003) by solving in the FD the following electrodynamic equation of a charge carrier subject to the action of an external electrical field $\boldsymbol{E}(\omega)$

$$
\sum_{p=0}^{2} m_{p}(i \omega)^{p} \boldsymbol{R}(\omega)=q \boldsymbol{E}(\omega)
$$

where $\omega$ is the angular frequency, $i$ is the imaginary unit, $q$ and $m_{2}$ represent the electric charge and the mass of the carrier, $m_{0}$ is an elastic-like coefficient accounting for recall effects, $m_{1}$ is a friction-like coefficient accounting for dissipative effects due, e.g., to collisions, and $\boldsymbol{R}(\omega)$ is the FT of the trajectory of the charge carrier.

Assuming for simplicity that the dispersive material contains only one species of charge carriers, indicating with $K$ the number of charge carriers per unit of volume, the following expression for the dispersive conductivity function $\sigma(w)$, called admittivity (Stoyer, 1976), has 
been derived (Patella, 2003)

$$
\sigma(\omega)=\frac{i \omega K q^{2}}{m_{0}+i \omega m_{1}-\omega^{2} m_{2}}
$$

Equation (1.2) is a simple IP model, which describes the behavior of a circuit-like cell made of an RCL series combination. It is equivalent to the Lorentz dispersion formula obtained as solution to the 2nd-order ordinary differential equation of harmonic oscillation (Balanis, 1989).

\section{Non-resonant positive IP model}

Let us consider now a system with two different species of charge carriers and put with $K_{j,} q_{j}$ and $m_{p, j}(p=0,1,2)$ the number per unit of volume, the electric charge and the passive coefficients, respectively, of the carriers of the $j$-th species $(j=1,2)$. Such a pair of ionic species can be the result of ionic dissociation of a salt dissolved in pore water. An interesting case to discuss is when one species $(j=1)$ is characterized by negligible recall and inertia terms, and the other species $(j=2)$ by only negligible inertia. This compound can, in fact, be used to explain the well-known IP electrode effect, caused by the presence of metallic or electronic conducting mineral particles occluding pore paths in rocks (Madden and Cantwell, 1967). Considering, for simplicity, a single-salt solution, only one ionic component, namely species 1 , is assumed to undergo redox reactions at the two opposite contact faces, in order for the electric current to flow from the solution through the metallic mineral. This is equivalent to claiming that only the passage of the reacting species 1 is allowed. The non-reactant ionic species 2, instead, after running short distances under the influence of the impressed field, must remain blocked along the frontal metallic face, thus giving rise to the IP effect. This simple compound can also explain the IP membrane effect in porous rocks containing dispersed clay particles in contact with an electrolytic solution (Madden and Cantwell, 1967), generally less strong than the IP electrode effect. Considering again a single-salt solution, species 1 and 2 would represent, respectively, the cations, carrying the electrical current fed by the external source, and anions, traveling, instead, short distances due to the blocking action by the negatively charged membranes (clay particles).

Referring to eq. (1.2), it is thus assumed that an elementary cell of a dispersive rock can behave like a two-branch parallel circuit, with a branch consisting of a single resistance and the other branch of a RC series sequence, i.e.

$$
\sigma^{(+)}(\omega)=\frac{K_{1} q_{1}^{2}}{m_{1,1}}+\frac{i \omega K_{2} q_{2}^{2}}{m_{0,2}+i \omega m_{1,2}}
$$

Putting

$$
\begin{gathered}
\sigma_{1}=\frac{K_{1} q_{1}^{2}}{m_{1,1}} \\
\sigma_{2}=\frac{K_{2} q_{2}^{2}}{m_{1,2}} \\
\gamma_{2}=\frac{m_{0,2}}{m_{1,2}}
\end{gathered}
$$

from eq. (2.1) we obtain $\sigma^{(+)}(\omega)$ as

$$
\sigma^{(+)}(\omega)=\frac{\sigma_{1} \gamma_{2}+i \omega\left(\sigma_{1}+\sigma_{2}\right)}{\gamma_{2}+i \omega}
$$

Equation (2.3) represents a simple non-resonant positive IP model, whose real and imaginary parts are qualitatively drawn versus frequency in fig. 1. The real part of the admittivity is always positive and its High-Frequency (HF) asymptote is located at a finite level higher than the Low-Frequency (LF) asymptote. The imaginary part, which is always positive, vanishes at both limits and presents a maximum in the inflexion point of the real part. The dispersion law expressed by eq. (2.3) is equivalent to the well known Debye model (Debye, 1928).

Generalizing, for each elementary cell either a parallel or a series combination of $N$ twobranch circuits can be considered. For a parallel combination, the admittivity takes the form

$$
\sigma^{(+)}(\omega)=\sum_{n=1}^{N} \frac{\sigma_{1 n} \gamma_{2 n}+i \omega\left(\sigma_{1 n}+\sigma_{2 n}\right)}{\gamma_{2 n}+i \omega}
$$

and for a series combination the dispersive resistivity function $\rho^{(+)}(\omega)$, called impedivity 


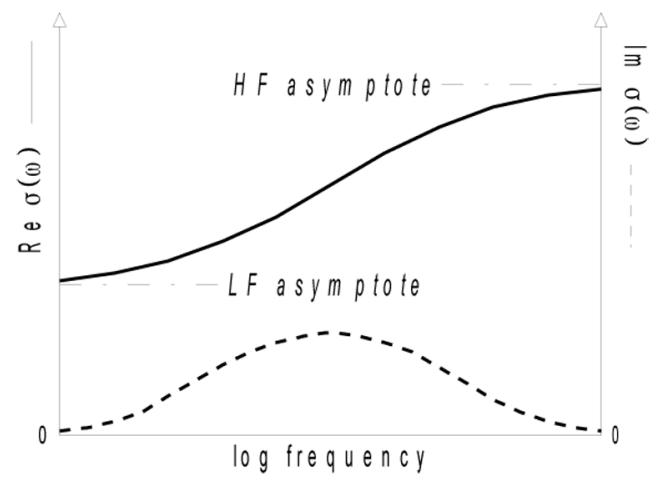

Fig. 1. Sketch diagram of the non-resonant positive IP model in FD.

(Patella, 1987), takes the form

$$
\rho^{(+)}(\omega)=\sum_{n=1}^{N} \frac{\gamma_{2 n}+i \omega}{\sigma_{1 n} \gamma_{2 n}+i \omega\left(\sigma_{1 n}+\sigma_{2 n}\right)}
$$

Putting

$$
\begin{gathered}
\rho_{0}=\lim _{\mathrm{w} \rightarrow 0} \rho^{+}(\omega)=\sum_{n=1}^{N}\left(\frac{1}{\sigma_{1 n}}\right) \\
\tau_{n}=\frac{\sigma_{1 n}+\sigma_{2 n}}{\gamma_{2 n} \sigma_{1 n}} \\
\beta_{n}=\frac{\sigma_{2 n}}{\rho_{0} \gamma_{2 n} \sigma_{1 n}^{2}}
\end{gathered}
$$

equation (2.5) can be written in the following best known form (Patella, 2003)

$$
\rho^{(+)}(\omega)=\rho_{0}\left(1-\sum_{n=1}^{N} \frac{i \omega \beta_{n}}{1+i \omega \tau_{n}}\right) .
$$

However, it is the Cole-Cole (CC) model (Cole and Cole, 1941) that has reached the greatest popularity in IP analysis since the pioneering work by Pelton et al. (1978), who reported the CC impedivity function $\rho^{C C}(\omega)$ in the form

$$
\rho^{C C}(\omega)=\rho_{0}\left[1-m \frac{(i \omega \tau)^{c}}{1+(i \omega \tau)^{c}}\right]
$$

where $\tau \geq 0$ and $c \in[0,1]$ are heuristic parameters required to adapt eq. (2.8) to experimental data, and $m \in[0,1]$ is the chargeability parameter introduced by Seigel (1959).
The CC model, though considered an empirical law (Wait, 1982), can physically be interpreted as a continuous distribution of Debye dispersion terms (Pelton et al., 1983), and hence approximated by an expression like eq. 2.7 (Patella and Di Maio, 2003).

Following the theory developed by Patella (1987, 1993), the CC model has been included in the magnetotelluric (MT) method to study the distortions provoked by IP on 1D (Di Maio et al., 1991) and 2D (Mauriello et al., 1996) synthetic responses. IP effects in MT have been experimentally recognized in volcanic and geothermal areas (Patella et al., 1991; Coppola et al., 1993; Giammetti et al., 1996; Di Maio et al., 1997, 2000; Mauriello et al., 2000, 2004).

\section{Non-resonant negative IP model}

Negative IP effects in earth materials are observed when $|\sigma(\omega)|<\left|\sigma_{0}\right|$ for $\omega>0$. In literature, negative IP is considered a geometric effect (Bertin and Loeb, 1976) and it was modeled for a polarizable sphere immersed in a uniform non-polarizable half-space (Sumner, 1967; Wait, 1982) and for certain layered media (Nabighian and Elliot, 1976). Sumner (1976) maintains that positive IP effects are usually associated with negative IP in definite patterns, and that for most surveys, positive values are larger than negative. He warns that if these conditions are not seen in the data, there may well be equipment or coupling problems. Madden and Cantwell (1967) hint that negative IP effects can be caused by leakages between transmitting and receiving circuits, and Bertin and Loeb (1976) also mention inductive coupling, which is sometimes added to and sometimes subtracted from the IP.

In conclusion, negative IP in the geophysical literature is not considered a physical effect, i.e. an intrinsic physical property of earth materials. This is rather surprising if one considers, for instance, that negative dispersion is an intrinsic physical property of electrons in metals at wavelengths sensibly less than about $10^{-2} \mathrm{~cm}$ (Stratton, 1941). It is shown now that a non-resonant negative IP model can be easily derived in the frame of the theory exposed in Section 1. 
Let us consider again a system with two different species of charge carriers and discuss the case when both species consist of unbound charges and the first species $(j=1)$ has negligible inertia. Referring to eq. (1.2), it is thus assumed that an elementary cell of a dispersive rock can now behave like a two-branch parallel circuit, with a branch made of a single resistance and the other branch of a RL series combination, i.e.

$$
\sigma^{(-)}(\omega)=\frac{K_{1} q_{1}^{2}}{m_{1,1}}+\frac{K_{2} q_{2}^{2}}{m_{1,2}+i \omega m_{2,2}}
$$

Using eq. (2.2a) and eq. (2.2b) and putting

$$
\lambda_{2}=\frac{m_{2,2}}{m_{1,2}}
$$

eq. (3.1) is written as

$$
\sigma^{(-)}(\omega)=\frac{\left(\sigma_{1}+\sigma_{2}\right)+i \omega \lambda_{2} \sigma_{1}}{1+i \omega \lambda_{2}}
$$

Equation (3.3) represents a simple non-resonant negative IP model. The similarity with eq. (2.3) allows $\sigma^{(-)}(\omega)$ to be considered a reverse Debye model. Its real and imaginary parts are qualitatively drawn versus frequency in fig. 2. Again the real part of the admittivity is always positive, but now its LF asymptote is placed at a fi-

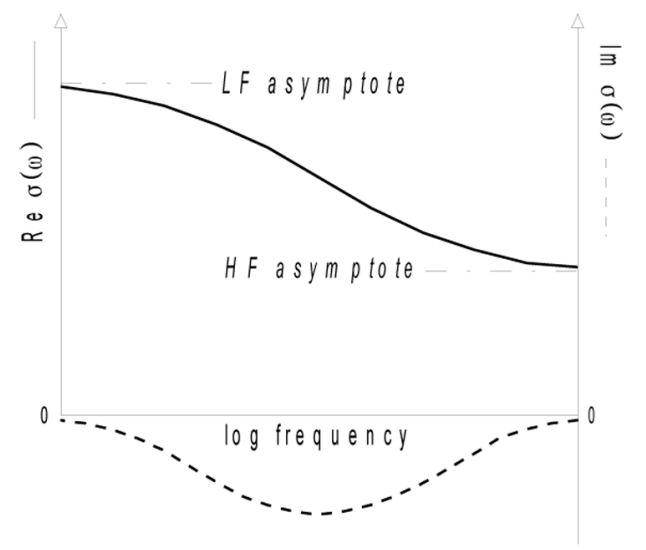

Fig. 2. Sketch diagram of the non-resonant negative IP model in FD. nite level higher than the HF asymptote. The imaginary part is now always negative, vanishes again for $\omega \rightarrow 0, \infty$ and presents a minimum in correspondence with the inflexion point of the real part.

Generalizing, for each elementary cell either a parallel or a series combination of $N$ twobranch circuits can again be considered. For a parallel combination, the admittivity takes the form

$$
\sigma^{(-)}(\omega)=\sum_{n=1}^{N} \frac{\left(\sigma_{1 n}+\sigma_{2 n}\right)+i \omega \lambda_{2 n} \sigma_{1 n}}{1+i \omega \lambda_{2 n}}
$$

while, for a series combination, the impedivity becomes

$$
\rho^{(-)}(\omega)=\sum_{n=1}^{N} \frac{1+i \omega \lambda_{2 n}}{\left(\sigma_{1 n}+\sigma_{2 n}\right)+i \omega \lambda_{2 n} \sigma_{1 n}} .
$$

\section{Resonant IP models}

Resonant IP effects are indeed included in the general solution given by eq. (1.2), hence a system with two different species of charge carriers is again considered by discussing the case when only the first species $(j=1)$ is characterized by negligible recall and inertia terms. It is thus assumed that an elementary cell of a dispersive rock now behaves like a two-branch parallel circuit, with a branch made of a single resistance and the other branch of a RLC series combination, i.e.

$\sigma^{(r)}(\omega)=\frac{K_{1} q_{1}^{2}}{m_{1,1}}+\frac{i \omega K_{2} q_{2}^{2}}{m_{0,2}+i \omega m_{1,2}-\omega^{2} m_{2,2}}$

which, recalling eqs.(2.2a) to (2.2c)) and eq. (3.2) can be rewritten as

$$
\sigma^{(r f)}(\omega)=\frac{\sigma_{1}\left(\gamma_{2}-\omega^{2} \lambda_{2}\right)+i \omega\left(\sigma_{1}+\sigma_{2}\right)}{\gamma_{2}+i \omega-\omega^{2} \lambda_{2}}
$$

Equation (4.2) represents a simple non-resonant flat IP model. Its real and imaginary parts are qualitatively drawn versus frequency in fig. 3 . The real part of the admittivity is again always positive, but its LF and HF asymptotes are now placed at the same finite level (flat asymptotic 


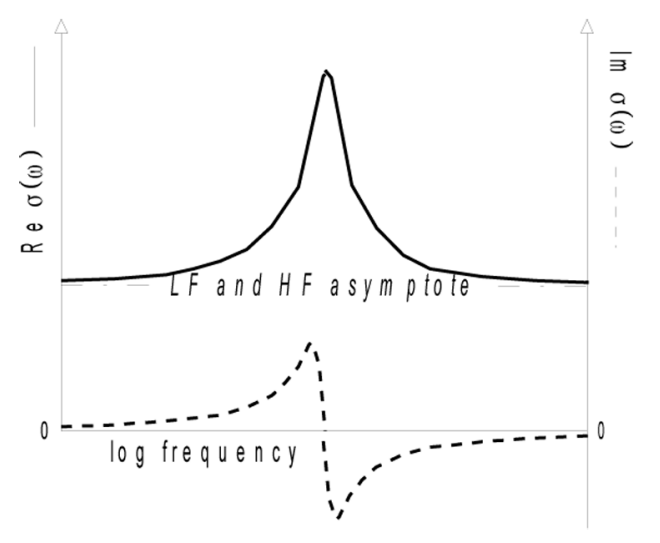

Fig. 3. Sketch diagram of the resonant flat IP model in FD.

line) and a bump appears with a maximum in correspondence with the resonance frequency $\omega=\left(\gamma_{2} / \lambda_{2}\right)^{1 / 2}$. The imaginary part goes to zero for $\omega \rightarrow 0, \infty$ and shows an undulation with the positive peak followed by the negative peak and crossing the $\omega$-axis in the resonance frequency. It is worth pointing out that a resonant effect can be considered a sequence of a positive and a negative IP effect.

Generalizing, for each elementary cell either a parallel or a series combination of $N$ twobranch circuits can as before be considered.

With no claim to be exhaustive, this survey on the IP physical modeling theory can now be concluded by considering resonance an additional phenomenon superimposed on either a positive or negative IP effect. It is thus assumed that an elementary cell of a dispersive rock can now contain three different ionic species $(j=1,2,3)$ and behave like a three-branch parallel circuit, with a branch made of a single resistance $(j=1)$, the second branch of either a RC or a RL series combination $(j=2)$, and the third branch by a RCL series combination $(j=3)$, i.e.

$$
\begin{aligned}
& \sigma^{(r+)}(\omega)=\frac{K_{1} q_{1}^{2}}{m_{1,1}}+\frac{i \omega K_{2} q_{2}^{2}}{m_{0,2}+i \omega m_{1,2}}+ \\
& +\frac{i \omega K_{3} q_{3}^{2}}{m_{0,3}+i \omega m_{1,3}-\omega^{2} m_{2,3}}
\end{aligned}
$$

for the resonant positive IP, and

$$
\begin{aligned}
& \sigma^{(r-)}(\omega)=\frac{K_{1} q_{1}^{2}}{m_{1,1}}+\frac{K_{2} q_{2}^{2}}{m_{1,2}+i \omega m_{2,2}}+ \\
& +\frac{i \omega K_{3} q_{3}^{2}}{m_{0,3}+i \omega m_{1,3}-\omega^{2} m_{2,3}}
\end{aligned}
$$

for the resonant negative IP.

Using the same symbolism as above, eq. (4.3) and eq. (4.4) can be given, respectively, as

$$
\begin{gathered}
\sigma^{(r+)}(\omega)=\frac{\sigma_{1} \gamma_{2}+i \omega\left(\sigma_{1}+\sigma_{2}\right)}{\gamma_{2}+i \omega}+\frac{i \omega \sigma_{3}}{\gamma_{3}+i \omega-\omega^{2} \lambda_{3}} \\
\sigma^{(r-)}(\omega)=\frac{\left(\sigma_{1}+\sigma_{2}\right)+i \omega \lambda_{2} \sigma_{1}}{1+i \omega \lambda_{2}}+\frac{i \omega \sigma_{3}}{\gamma_{3}+i \omega-\omega^{2} \lambda_{3}} .
\end{gathered}
$$

Figures 4 and 5 show qualitatively the behavior of the real and imaginary parts of the resonant positive IP model given by eq. (4.5) and the resonant negative IP model given by eq. (4.6). The result is the sum of the diagrams of the real and imaginary parts depicted in fig. 1 and fig. 2, respectively, plus the homologous diagrams in fig. 3, provided that a shift to the zero-level is applied to the common LF and HF asymptote of the real part.

The influence of the resonant positive IP

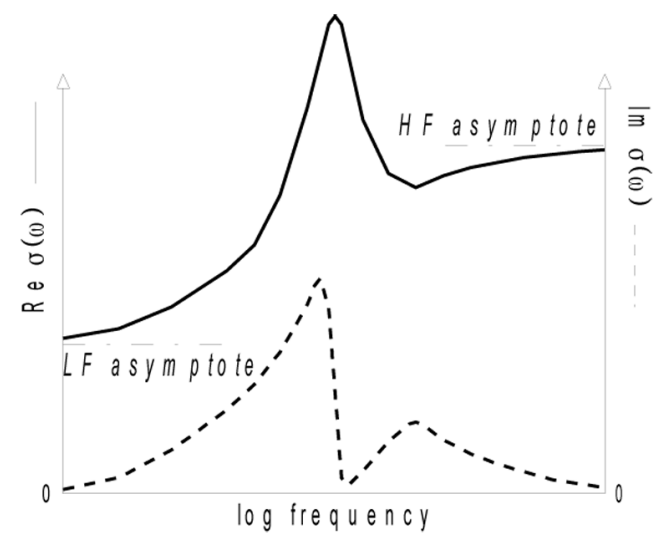

Fig. 4. Sketch diagram of the resonant positive IP model in FD. 


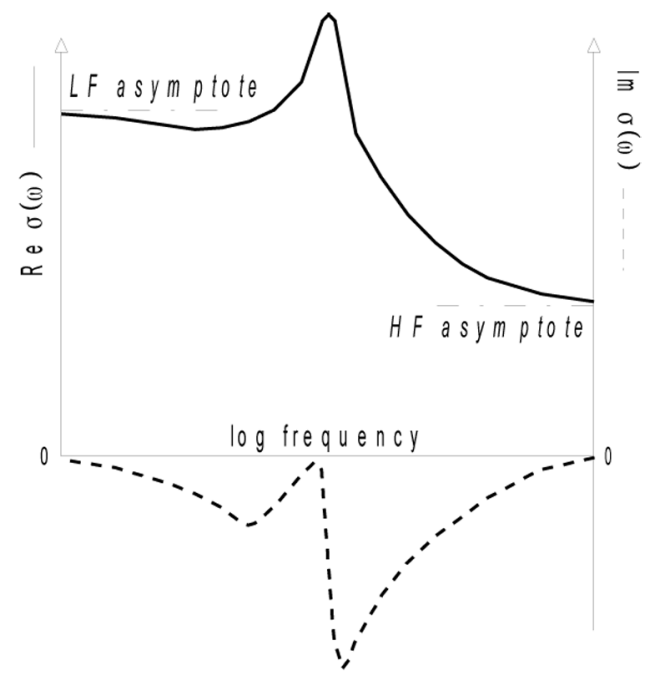

Fig. 5. Sketch diagram of the resonant negative IP model in FD.

phenomenon on transient EM methods has been studied in detail by Ageev and Svetov (1999) and Svetov and Ageev (1999). They adopted the $\mathrm{CC}$ model given in eq. (2.8), by arguing that for small chargeability values its validity can be extended to values of $c \in] 1,2[$ and that within this interval of $c$ resonance effects can be modeled.

\section{Conclusions}

It has been shown that IP phenomena in rocks can be described using conductivity dispersion laws derived as solutions to a linear 2nd-order differential equation defining the motion of a charged particle immersed in an external electrical field. Five dispersion laws have been discussed, namely:

1) the non-resonant positive IP model, which leads to the classical Debye dispersion law and by extension to the Cole-Cole law, largely used in current practice;

2) the non-resonant negative IP model, which introduces the possibility of explaining negative chargeability values, known in metals at high frequencies, also as an intrinsic physical effect in some earth materials;

3) the resonant flat, positive or negative IP models, which explain the presence of peak effects at specific frequencies superimposed on flat, positive or negative dispersion spectra.

Though all the derived dispersion models are related to the physical parameters regulating the motion of charge carriers under the influence of an external electric field, it must once again be remarked that distinguishing ionic species in earth materials by IP spectra still remains a difficult task. However, decrypting field measurements by one of the derived IP models may be very useful in some exploration problems.

To conclude, it is worth recalling that nonresonant positive IP responses constitute by far the largest majority of effects observed directly by the IP method in mineral, oil and groundwater investigations, and, indirectly by the MT method, also in geothermal areas. A wealth of literature exists on this topic, covering the last six decades almost continuously, since the pioneering intensive works started in the late 40's of the last century. Less known in literature are resonant IP responses, mostly studied by Russian researchers, of which good examples are reported in Safonov et al. (1996). Finally, regarding the negative IP effect as a true physical property of rocks, there is not yet any documentation firmly attesting such a possibility. Nevertheless, it is suspected that in environmental applications, for instance, the observation of nearsurface negative IP effects may be a useful indicator of the presence of massive ionic contaminants in conductive sediments.

\section{REFERENCES}

AgeEv, V.V. and B.S. Svetov (1999): The influence of rock polarizability on electromagnetic soundings, Izvestia RAS, Physics of the Solid Earth, 35, 16-24.

BALANIS, C.A. (1989): Advanced Engineering Electromagnetics (J. Wiley \& Sons, New York).

Bertin, J. and J. Loeb (1976): Experimental and Theoretical Aspects of Induced polarization (Gebrüder-Bornträger, Berlin), 2 vols.

Cole, K.S. and R.H. Cole (1941): Dispersion and Absorption in dielectrics, J. Chem. Phys., 9, 341-351.

Coppola, B., R. Di Maio, I. Marini, A. Merla, D. PatelLA, G. Pulelli, F.M. Rossi and A. Siniscalchi (1993): Study of the Simplon area geothermal anomaly in the 
frame of a transalpine deep railway tunnel feasibility projectin, in Underground Transportation Infrastructures, Proc. of an Intern. Conf. of the Association Française des Travaux en Souterrain, edited by J.L. REITH (Balkema, Rotterdam), Toulon, 1993, 93-102.

Debye , P. (1928): Polar Molecules (Chemical Catalogue Company, New York).

Di Maio, R., D. Patella and A. Siniscalchi (1991: Sul problema del riconoscimento di uno strato elettricamente polarizzabile mediante misure magnetotelluriche (in Italian), in Atti del II Convegno di Geomagnetismo ed Aeronomia, edited by A. MELONI and B. ZOLESI (Istituto Nazionale di Geofisica e Vulcanologia, Roma), 239-250.

Di Maio, R., P. Mauriello, D. Patella, Z. Petrillo, S. Piscitelli, A. Siniscalchi and M. Veneruso (1997): Self-potential, geoelectric and magnetotelluric studies in Italian active volcanic areas, Ann. Geofis., XL (2), 519-537.

Di Maio, R., D. Patella, Z. Petrillo, A. Siniscalchi, G. Cecere and P. De Martino (2000): Application of electric and electromagnetic methods to the study of the Phlegrean Fields caldera, Ann. Geofis., 43 (2), 375-390.

Fink, J.B., E.O. McAlister, B.K. Sternberg, W.G. WIEDUWILT and S.H. WARD (Editors) (1990): Induced polarization: applications and case histories, in Investigations in Geophysics, Society of Exploration Geophysicists, Tulsa, Oklahoma, vol. 4, pp. 414.

Giammetti, S., D. Patella, A. Siniscalchi and A. TraMACERE (1996): The Siena Graben: Combined interpretation of DES and MT soundings, Ann. Geofis., XXXIX (1), 189-200.

Madden, T.R. and T. CANTwEll (1967): Induced polarization, a review, in Mining Geophysics, Society of Exploration Geophysicists, Tulsa, Oklahoma, vol. 2, 373-400.

Mauriello, P., D. Patella and A. Siniscalchi (1996): The magnetotelluric response over two-dimensional media with resistivity frequency dispersion, Geophys. Prosp., 44, 789-818.

Mauriello, P., D. Patella, Z. Petrillo and A. SinisCALCHI (2000): An integrated magnetotelluric study of the Mt. Etna volcanic structure, Ann. Geofis., 43 (2), 325-342.

Mauriello, P., D. Patella, Z. Petrillo, A. Siniscalchi, T. IUliano and C. Del Negro (2004): A geophysical study of the Mt. Etna volcanic area, in Mt. Etna: Volcano Laboratory, edited by A. BONACCORSO, S. CALVARI, M. Coltelli, C. Del Negro and S. FAlsaperla, American Geophysical Union, Geophysical Monograph Series, 143, 273-291.

Nabighian, M.N., and C.L. Elliot (1976): Negative induced polarization effects from layered media, Geophysics, 41, 1235-1255.
PATELla, D. (1987): Tutorial: Interpretation of magnetotelluric measurements over an electrically dispersive onedimensional earth, Geophys. Prosp., 35, 1-11.

PATElla, D. (1993): I principi metodologici della magnetotellurica su mezzi generalmente dispersivi (in Italian), Ann. Geofis., XXXVI (5/6), 147-160.

Patella, D. (2003): On the role of the $J-E$ constitutive relationship in applied geoelectromagnetism, Ann. Geophys., 46, 589-597.

Patella, D. and R. Di Maio (1989): On the analysis of Cole-Cole relaxations transients in the induced polarization prospecting method, in Inverse Modeling in Exploration Geophysics, edited by A. VoGEL, R. Gorenflo, B. Kummer and C.O. OFoegbu (F. Vieweg \& Sohn, Braunschweig/Wiesbaden), 205-219.

Patella, D., A. Tramacere, R. Di Maio and A. SinisCALCHI (1991): Experimental evidence of resistivity frequency-dispersion in magnetotellurics in the Newberry (Oregon), Snake River Plain (Idaho) and Campi Flegrei (Italy) volcano-geothermal areas, J. Volcanol. Geoth. Res., 48, 61-75.

Pelton, W.H., S.H. Ward, P.G. Hallof, W.R. Sill and P.H. NELSON (1978): Mineral discrimination and removal of inductive coupling with multi-frequency IP, Geophysics, 43, 588-603.

Pelton, W.H., W.R. SiLl and B.D. Smith (1983): Interpretation of complex resistivity and dielectric data. Part I, Geophys. Trans., 29, 297-330.

Safonov, A.S., I.A. Mushin, E.S. Kiselev and A.S. GoRYUNOV (1996): A structural-formation model as the physical-geological basis for high-resolution electroprospecting, Geofizika, 2, 12-23.

SEIGEL, H.O. (1959): Mathematical formulation and type curves for induced polarization, Geophysics, 24, 547565.

StOYeR, C.H. (1976): Consequences of induced polarization in magnetotelluric interpretation, Pure Appl. Geophys., 114, 435-449.

StratTon, J. (1941): Electromagnetic Theory (McGrawHill, New York).

SuMnER, J.S. (1967): The problem of negative IP anomalies, in Proc. of the Symposium on Induced Electrical Polarization, Engineering Geoscience, Department of Mineral, Technical University of California, Berkeley, 32-50.

Sumner, J.S. (1976): Principles of Induced Polarization for Geophysical Exploration (Elsevier, Amsterdam).

Svetov, B.S. and V.V. AgeEv, (1999): High resolution electromagnetic methods and low fequency dispersion of rock conductivity, Ann. Geofis., 42 (4), 699-713.

WAIT, J.R. (Editor) (1959): Overvoltage Research and Geophysical Applications (Pergamon, Oxford).

WAIT, J.R. (1982): Geo-electromagnetism (Academic Press, New York). 